\title{
A Cyclodextrin-Modified Ketoester for Stereoselective Epoxidation of Alkenes
}

\author{
Wing-Kei Chan, Wing-Yiu Yu, Chi-Ming Che, and Man-Kin Wong* \\ Department of Chemistry and Open Laboratory of Chemical Biology of the Institute of \\ Molecular Technology for Drug Discovery and Synthesis, The University of Hong Kong, \\ Pokfulam Road, Hong Kong, China \\ mkwong@hkusua.hku.hk
}

\section{SUPPORTING INFORMATION}

\section{Table of Contents}

Characterization data of terpenes $(S)-\mathbf{3 b} \mathbf{d}$

S2 S3

References of epoxides $4 \mathbf{a} \mathbf{c}$ and limonene epoxides

S3

Characterization data of $\mathbf{4 d}$

S4

${ }^{1} \mathrm{H}$ NMR spectra of diastereomeric mixtures of terpene epoxides

S5 S7

${ }^{1} \mathrm{H}$ NMR spectra of enantiomeric mixtures of epoxides

S8 S13

References for the determination of enantiomeric excesses of epoxides

S14

${ }^{1} \mathrm{H}$ NMR spectrum of 2

S15

${ }^{13} \mathrm{C}$ NMR spectrum of 2

S16

${ }^{1} \mathrm{H}$ NMR spectrum of $\mathbf{4 d}$

S17

${ }^{13} \mathrm{C}$ NMR spectrum of $\mathbf{4 d}$

S18

Partial contour plot of the ROESY spectrum of the $2 / 3 \mathbf{b}$ complex

S19 


\section{(I) Characterization Data of Terpenes $(S)-3 \mathrm{~b}$ d}

Terpenes $(S)$-3b-d were prepared from $(S)$ - -terpineol $(\mathbf{3 a})\left([\alpha]_{\mathrm{D}}{ }^{20} 79.1 \quad(\mathrm{c}=20, \mathrm{EtOH})\right)$.

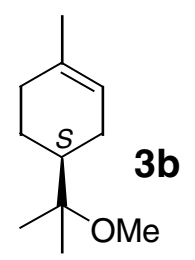

${ }^{1} \mathrm{H}$ NMR (300 MHz, CDCl $)_{3} \quad 5.39$ (br s, 1H), $3.18(\mathrm{~s}, 3 \mathrm{H}), 1.672 .02(\mathrm{~m}, 6 \mathrm{H}), 1.65(\mathrm{~s}, 3 \mathrm{H})$, $1.161 .30(\mathrm{~m}, 1 \mathrm{H}), 1.10(\mathrm{~s}, 3 \mathrm{H}), 1.09(\mathrm{~s}, 3 \mathrm{H}) ;{ }^{13} \mathrm{C} \mathrm{NMR}\left(75.47 \mathrm{MHz}, \mathrm{CDCl}_{3}\right) \quad 133.99,120.84$, 76.63, 48.69, 41.48, 31.08, 26.84, 23.91, 23.35, 22.33, 21.84. (ref: Crivello, J. V.; Liu, S. Chem. Mater. 1998, 10, 3724.)

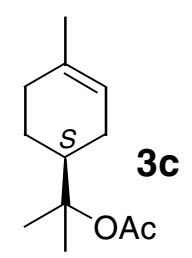

${ }^{1} \mathrm{H}$ NMR (300 MHz, $\left.\mathrm{CDCl}_{3}\right) \quad 5.37$ (br s, 1H), 1.712 .19 (m, 6H), 1.94 (m, 3H), 1.64 (s, 3H), $1.44(\mathrm{~s}, 3 \mathrm{H}), 1.41(\mathrm{~s}, 3 \mathrm{H}), 1.231 .36(\mathrm{~m}, 1 \mathrm{H}),{ }^{13} \mathrm{C} \mathrm{NMR}\left(75.47 \mathrm{MHz}, \mathrm{CDCl}_{3}\right) \quad$ 170.47, 133.94, 120.29, 84.82, 42.55, 30.87, 26.35, 23.84, 23.31, 23.10, 22.47. (ref: Hirata, T.; Izumi, S.; Ekida, T.; Suga, T. Bull. Chem. Soc. Jpn. 1987, 60, 289.)

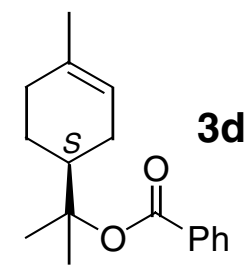


${ }^{1} \mathrm{H}$ NMR (300 MHz, CDCl $) \quad 7.978 .00(\mathrm{~m}, 2 \mathrm{H}), 7.347 .55$ (m, 3H), $5.40(\mathrm{br} \mathrm{s}, 1 \mathrm{H}), 1.872 .18$ $(\mathrm{m}, 6 \mathrm{H}), 1.67(\mathrm{~s}, 3 \mathrm{H}), 1.60(\mathrm{~s}, 3 \mathrm{H}), 1.58(\mathrm{~s}, 3 \mathrm{H}), 1.381 .54(\mathrm{~m}, 1 \mathrm{H}) ;{ }^{13} \mathrm{C}$ NMR $(75.47 \mathrm{MHz}$, $\left.\mathrm{CDCl}_{3}\right) \quad 165.30,133.98,132.38,132.09,129.36,128.19,120.35,85.48,43.19,30.94,26.48$, 24.02, 23.45, 23.41, 23.30; $[\alpha]_{\mathrm{D}}{ }^{20} 44$ (c = 4, EtOAc). (ref: Ravindranath, B.; Srinivas, P. Tetrahedron 1984, 40, 1623.)

\section{(II) References of Epoxides 4a c and Limonene epoxides}

\begin{tabular}{|l|l|l|}
\hline & Copperman, H. L.; Hallcher, R. C.; Riehl, A.; Carlson, R. M.; \\
\hline
\end{tabular}


(III) Characterization Data of $4 d$

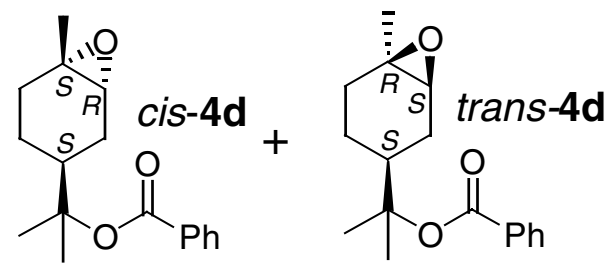

Colorless liquid; analytical TLC (silica gel 60) (10\% EtOAc in hexane), $\mathrm{R}_{f}=0.41 ;{ }^{1} \mathrm{H}$ NMR $(300$ $\left.\mathrm{MHz}, \mathrm{CDCl}_{3}\right) 7.957 .99(\mathrm{~m}, 2 \mathrm{H}), 7.397 .54(\mathrm{~m}, 3 \mathrm{H}), 3.11$ (br s, $\left.1 / 2 \quad 1 \mathrm{H}\right), 3.01$ (d, J= $5 \mathrm{~Hz}, 1 / 2$ 1H), $1.662 .33(\mathrm{~m}, 6 \mathrm{H}), 1.58(\mathrm{~s}, 3 \mathrm{H}), 1.55(\mathrm{~s}, 1 / 2 \quad 3 \mathrm{H}), 1.50(\mathrm{~s}, 1 / 2 \quad 3 \mathrm{H}), 1.35(\mathrm{~s}, 1 / 2 \quad 3 \mathrm{H})$, $1.32(\mathrm{~s}, 1 / 2 \quad 3 \mathrm{H}) ;{ }^{13} \mathrm{C}$ NMR (75.47 MHz, $\left.\mathrm{CDCl}_{3}\right) \quad$ 165.61, 165.47, 132.55, 132.45, 132.02, $131.78,129.38,129.33,128.26,128.22,85.26,84.65,60.99,59.12,57.68,57.52,41.59,39.63$, $30.76,29.42,26.88,25.53,24.38,23.48,23.42,23.30,22.85,22.82,20.28$; IR (KBr) $1712 \mathrm{~cm}^{1}$; FABMS $m / z, 275\left(\mathrm{M}^{+}, 18\right)$; HRMS (EI) for $\mathrm{C}_{10} \mathrm{H}_{16} \mathrm{O}\left(\mathrm{M}^{+} \mathrm{PhCOOH}\right)$, calcd 152.1201, found 152.1195. 
(IV) ${ }^{1} \mathrm{H}$ NMR Spectra of Diastereomeric Mixtures of Terpene Epoxides

Table 1, entry 1

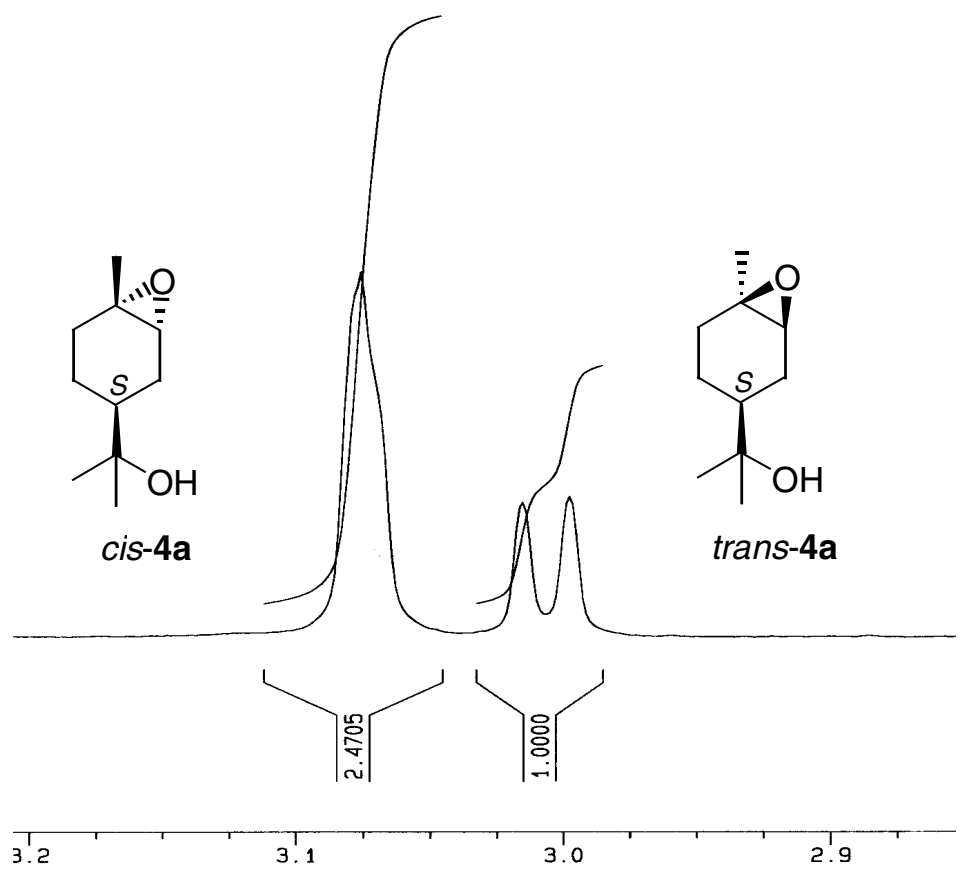

Table 1, entry 6

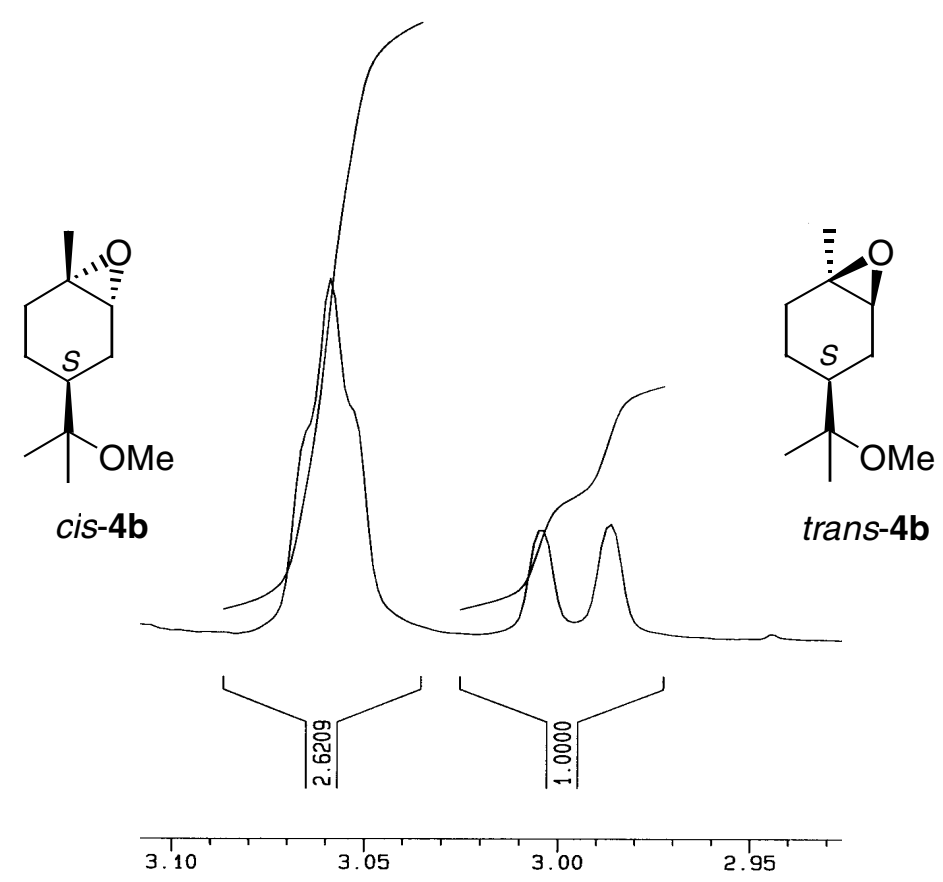


Table 1, entry 9

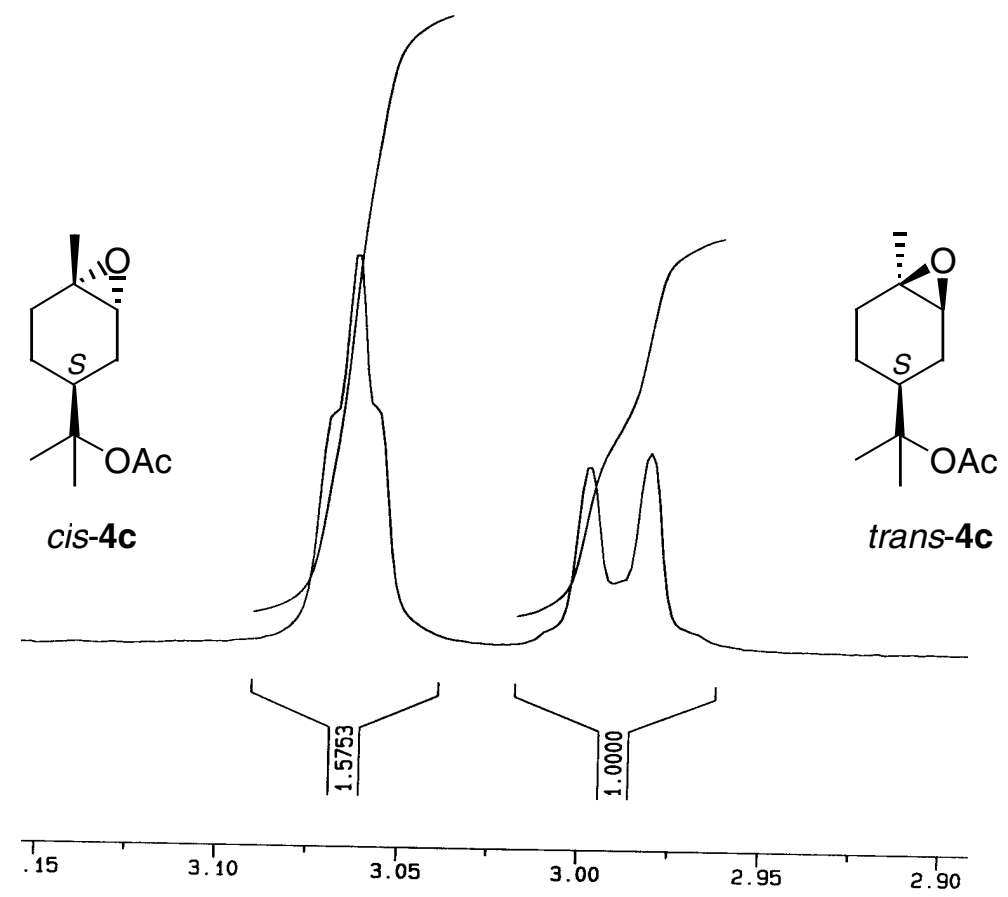

Table 1 , entry 12

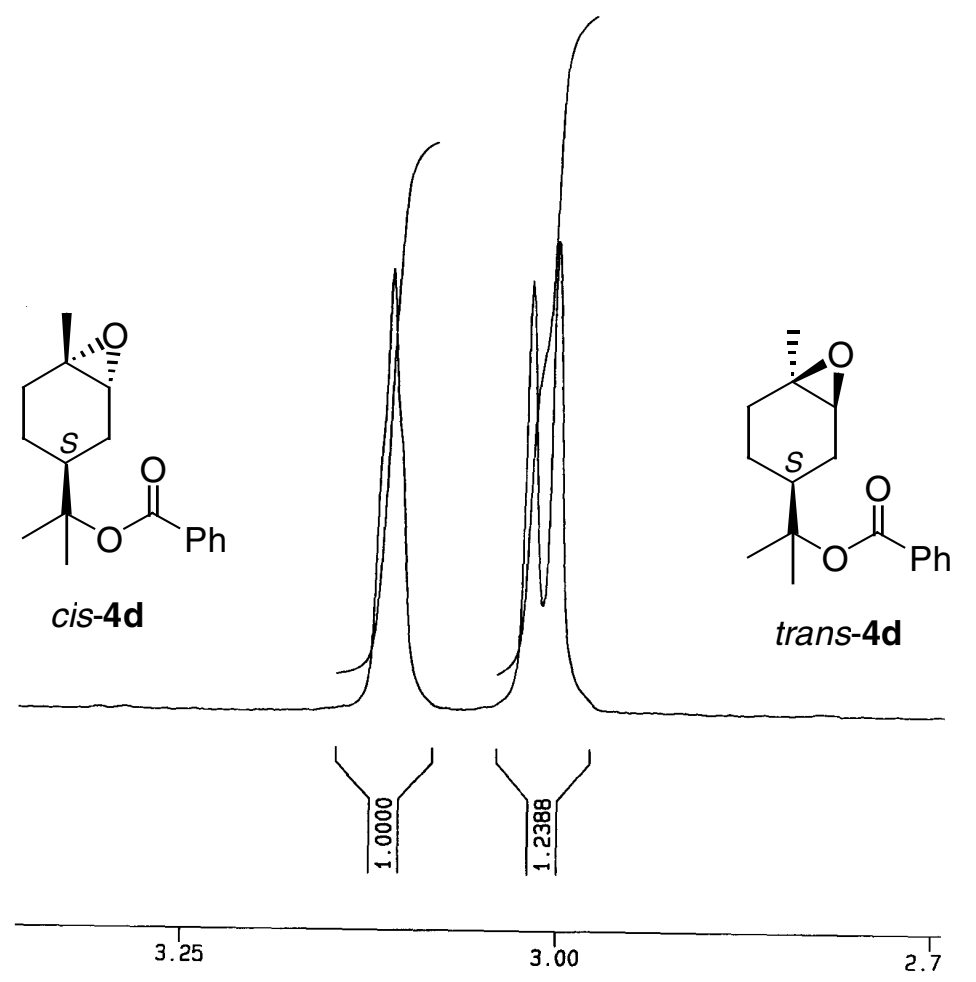


Table 2, entry 1

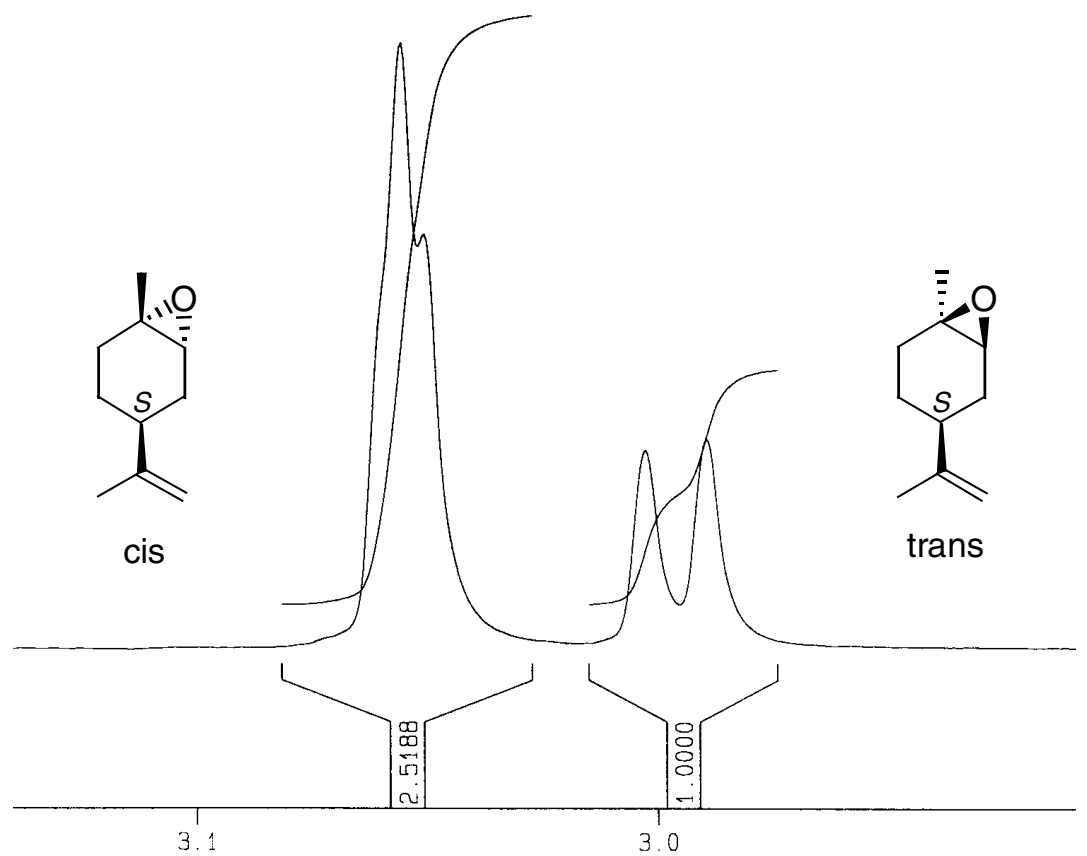

Table 2, entry 4

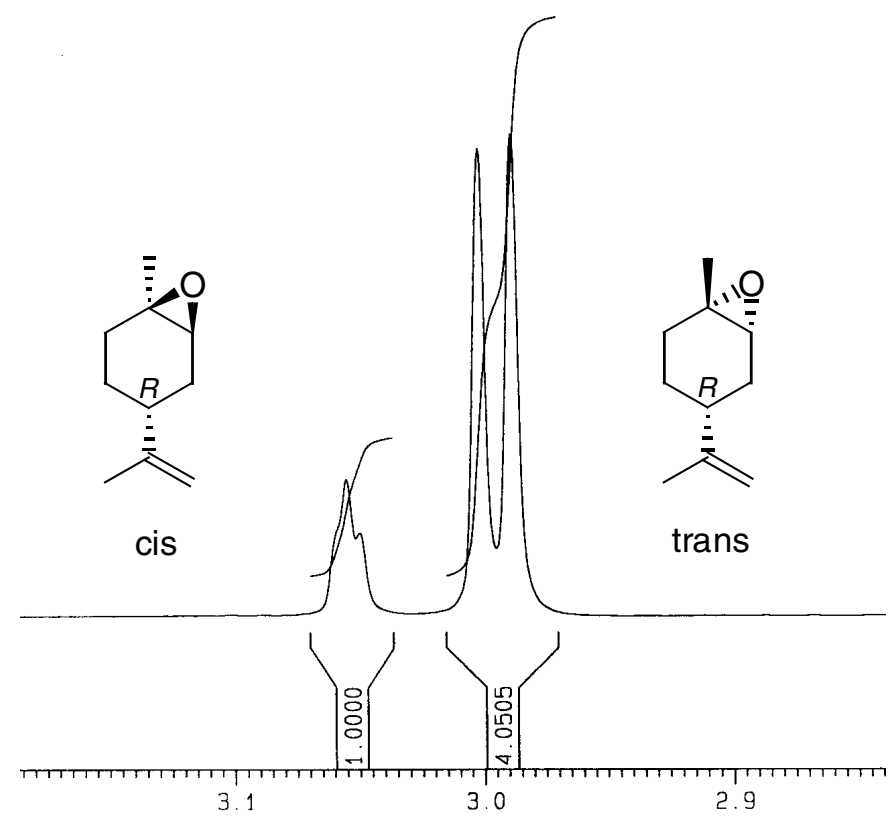




\section{(V) ${ }^{1}$ H NMR Spectra of Enantiomeric Mixtures of Epoxides}

Enantiopurities of the chiral epoxides were determined by ${ }^{1} \mathrm{H}$ NMR spectroscopy using Eu(hfc $)_{3}$ (Aldrich Cat. No. 16,474-7) as chiral shift reagent. The percentage of enantiomeric excess (\%ee) was evaluated on the basis of the integration ratio of the corresponding resolved peak areas. The spectra of each epoxide in both racemic and enantiomerically enriched forms are provided on the following pages.

Table 3, entry 4

${ }^{1} \mathrm{H}$ NMR Conditions<smiles>Pc1ccccc1</smiles>
Chiral shift reagent: $\mathrm{Eu}(\mathrm{hfc})_{3}$
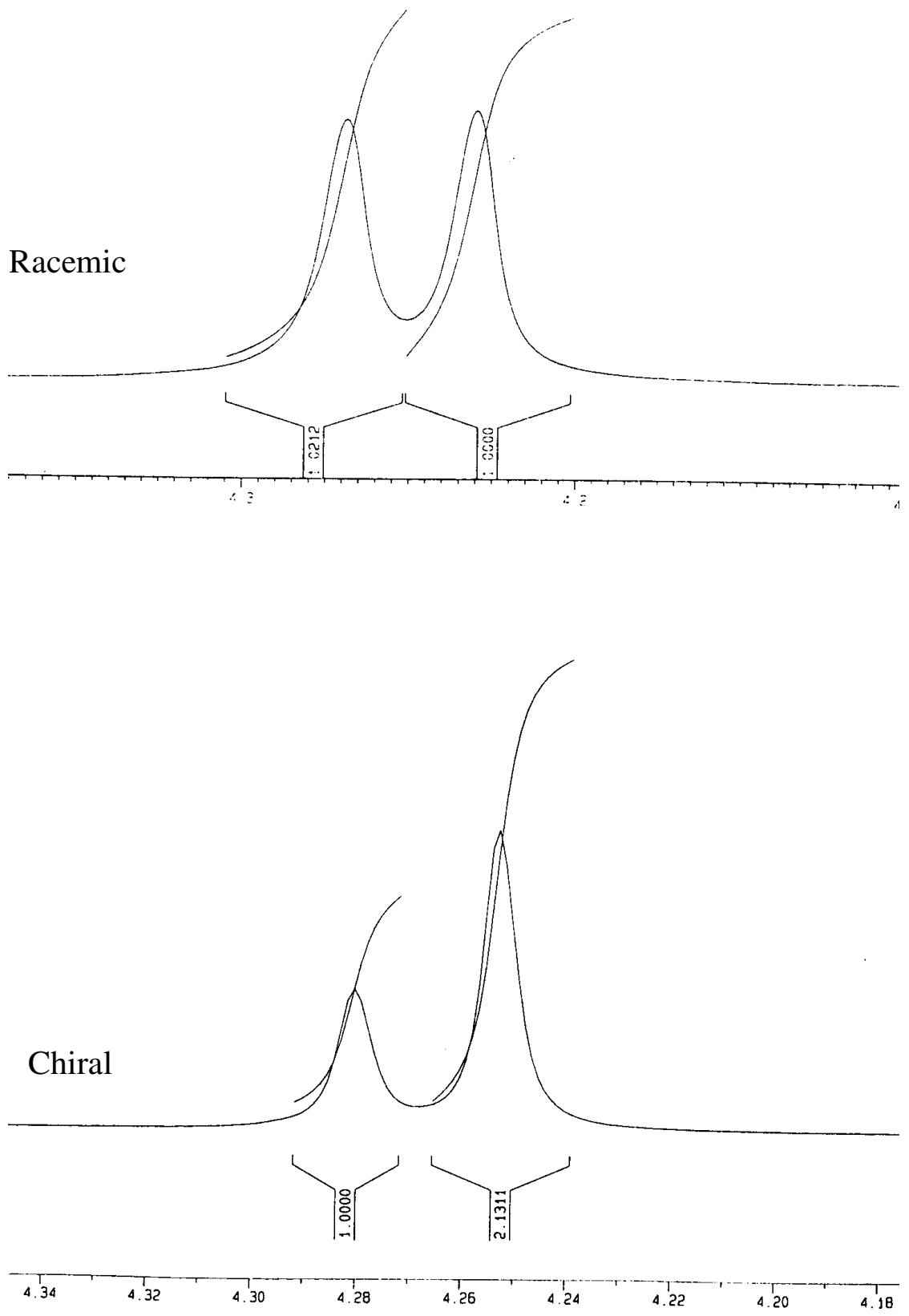
Table 3 , entry 5

${ }^{1} \mathrm{H}$ NMR Conditions

Chiral shift reagent: $\mathrm{Eu}(\mathrm{hfc})_{3}$
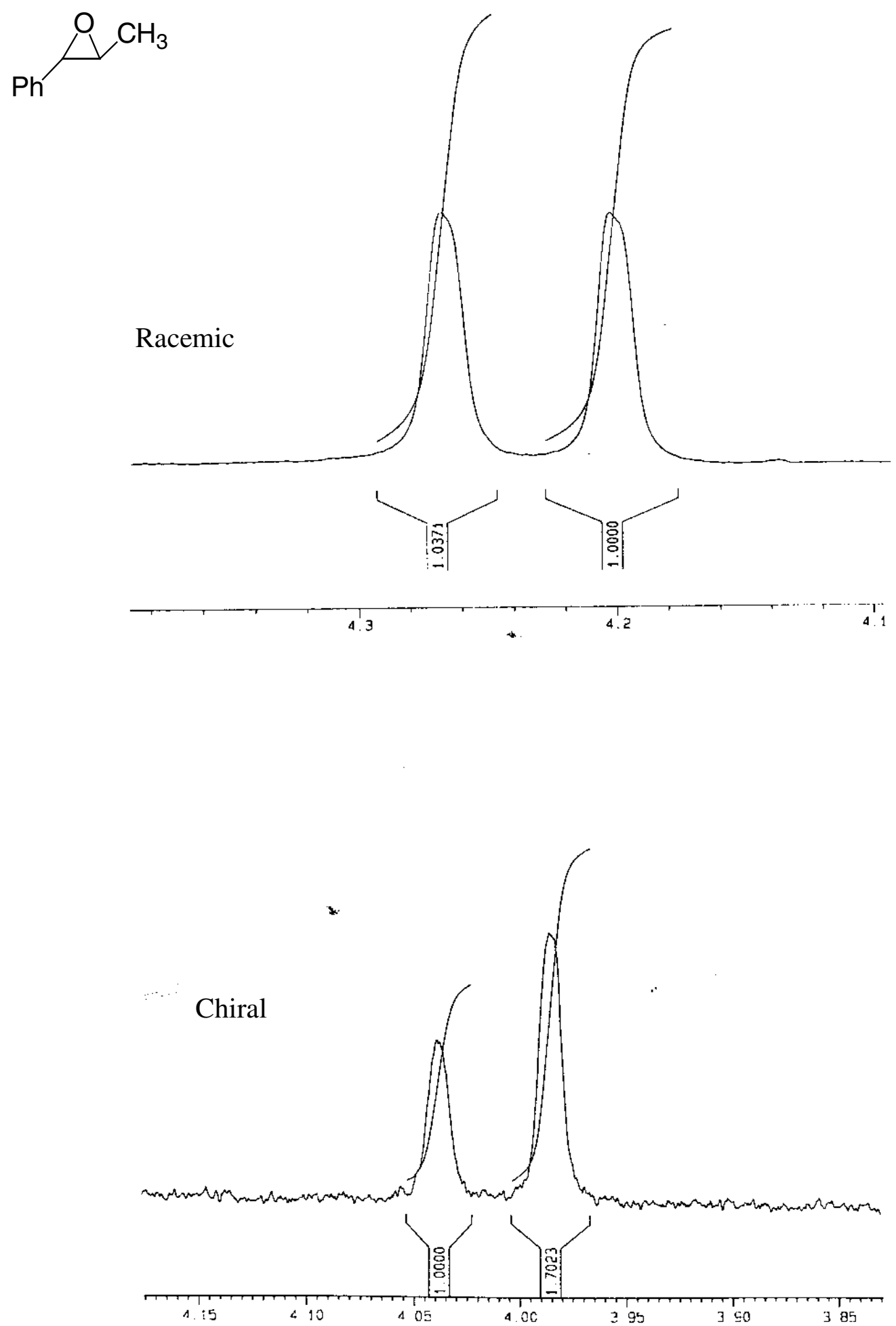
Table 3, entry 6

${ }^{1} \mathrm{H}$ NMR Conditions

Chiral shift reagent: $\mathrm{Eu}(\mathrm{hfc})_{3}$
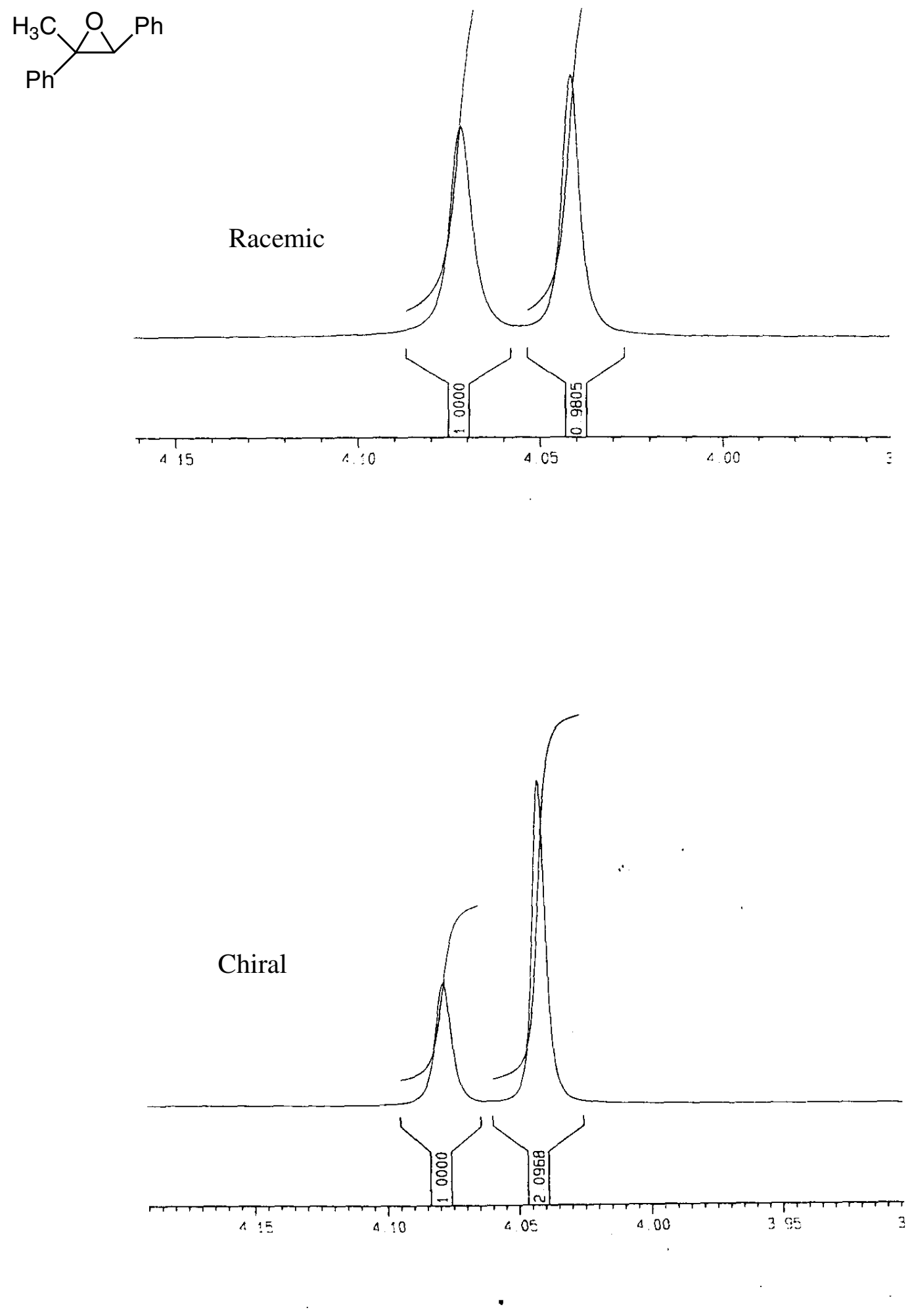
Table 3, entry 7

${ }^{1} \mathrm{H}$ NMR Conditions

Chiral shift reagent: $\mathrm{Eu}(\mathrm{hfc})_{3}$
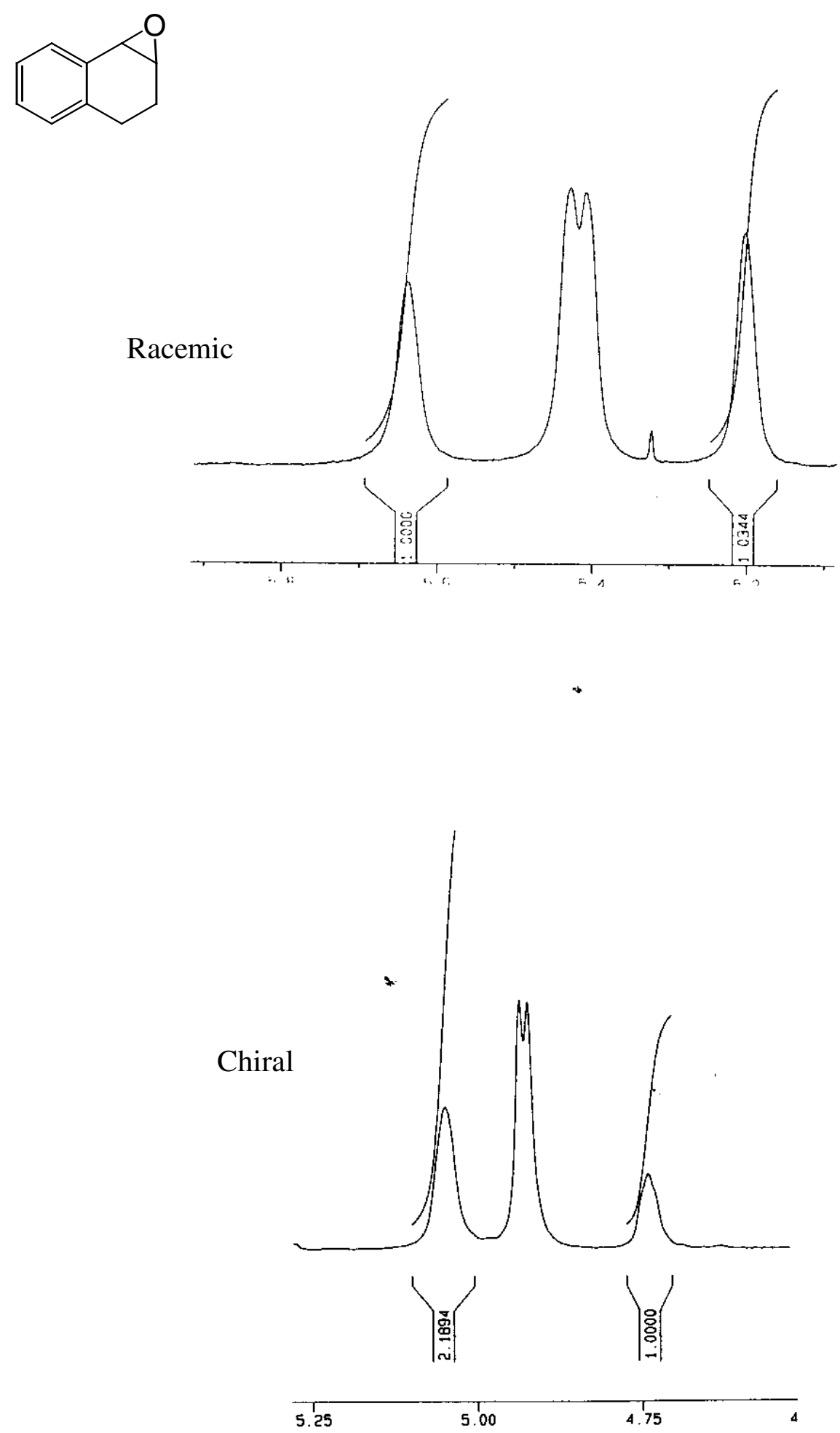
Table 3, entry 9

${ }^{1} \mathrm{H}$ NMR Conditions

Chiral shift reagent: $\mathrm{Eu}(\mathrm{hfc})_{3}$<smiles>Clc1ccc(C2CO2)cc1</smiles>
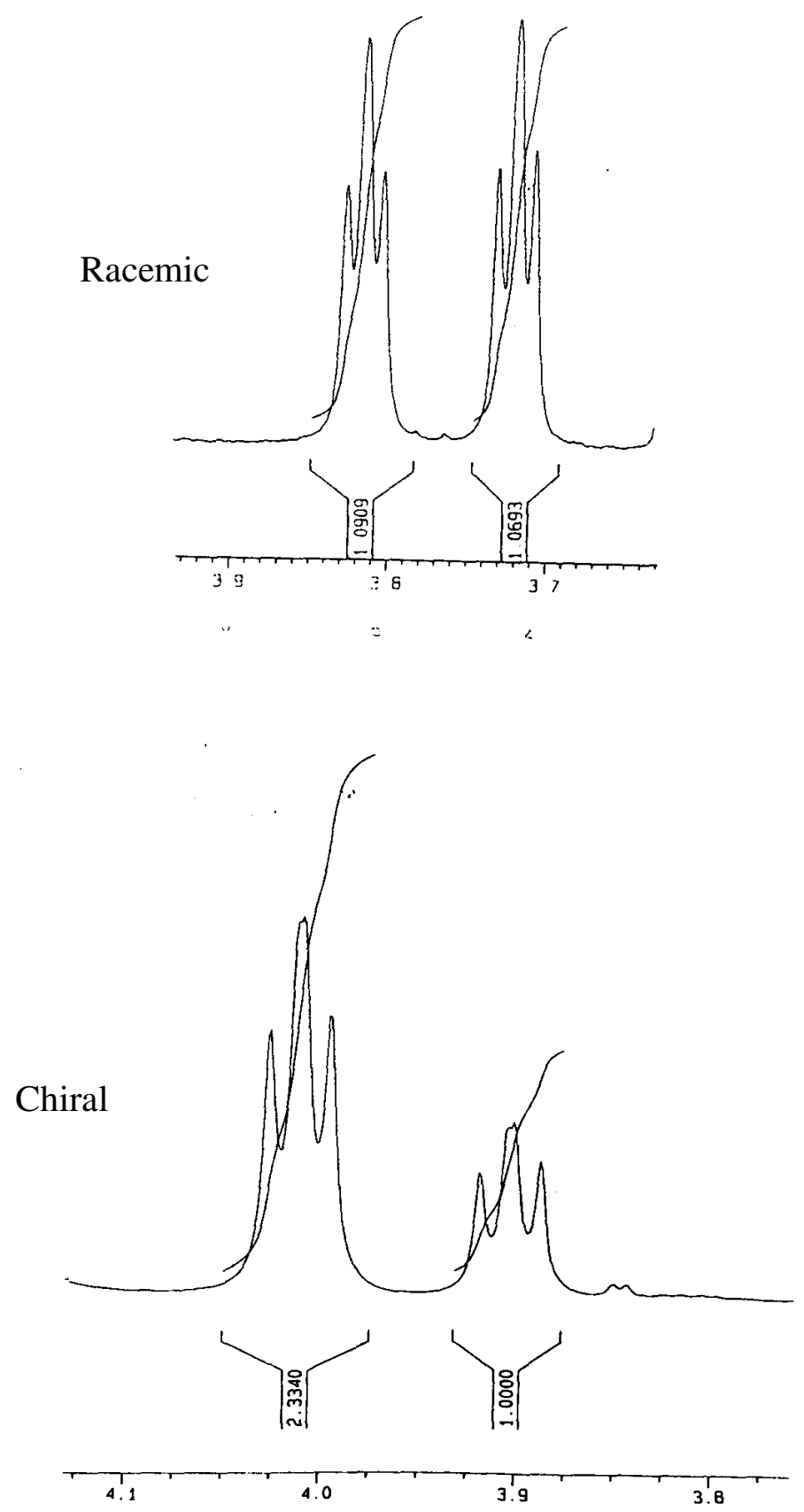
Table 3 , entry 10

${ }^{1} \mathrm{H}$ NMR Conditions

Chiral shift reagent: $\mathrm{Eu}(\mathrm{hfc})_{3}$
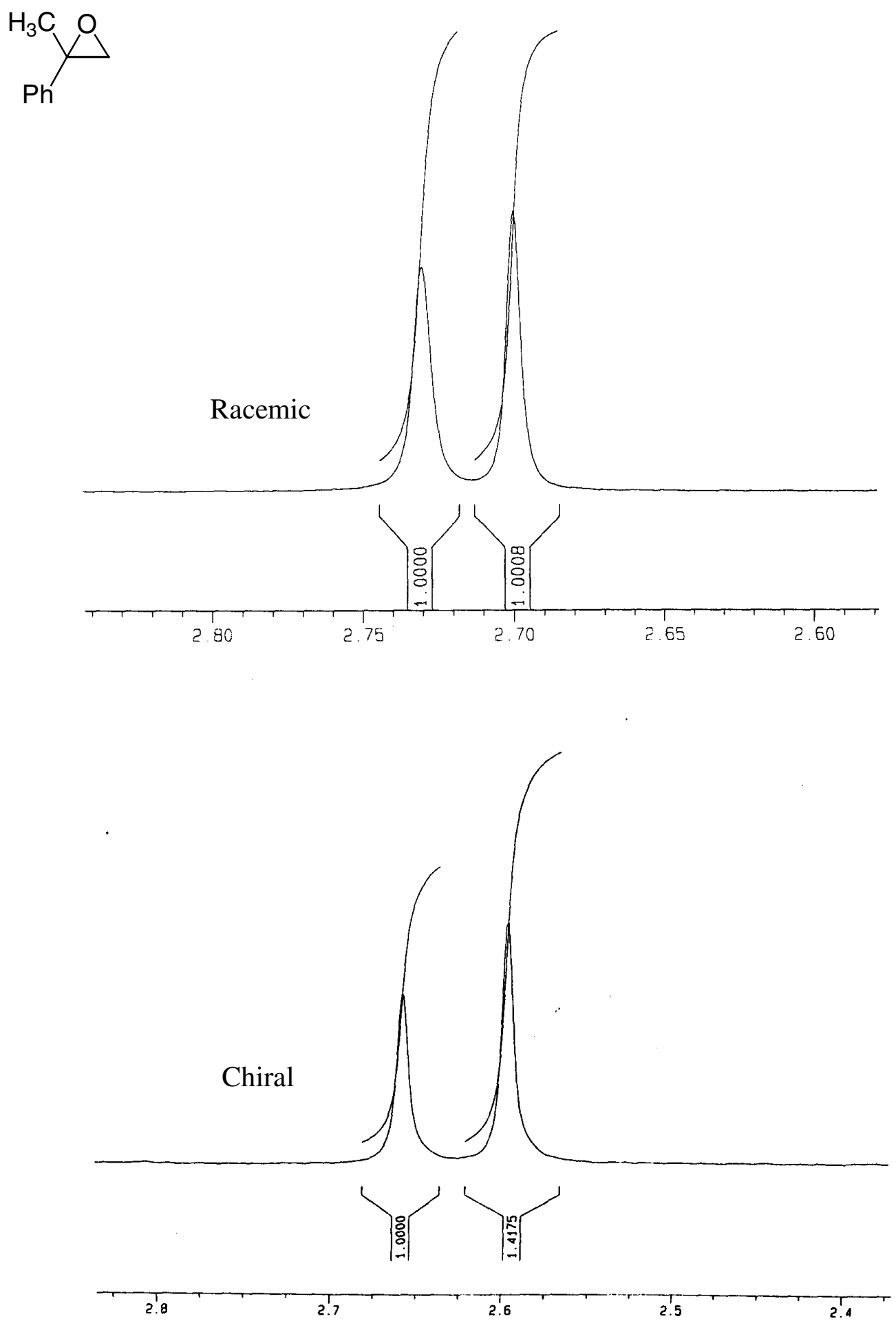
References for the determination of enantiomeric excesses of epoxides:

\begin{tabular}{|l|l|} 
Wang, Z. X.; Tu, Y.; Frohn, M.; Shi, Y. J. Org. Chem. 1997, 62, 2328. \\
\hline Wang, Z. X.; Tu, Y.; Frohn, M.; Shi, Y. J. Org. Chem. 1997, 62, 2328.
\end{tabular}




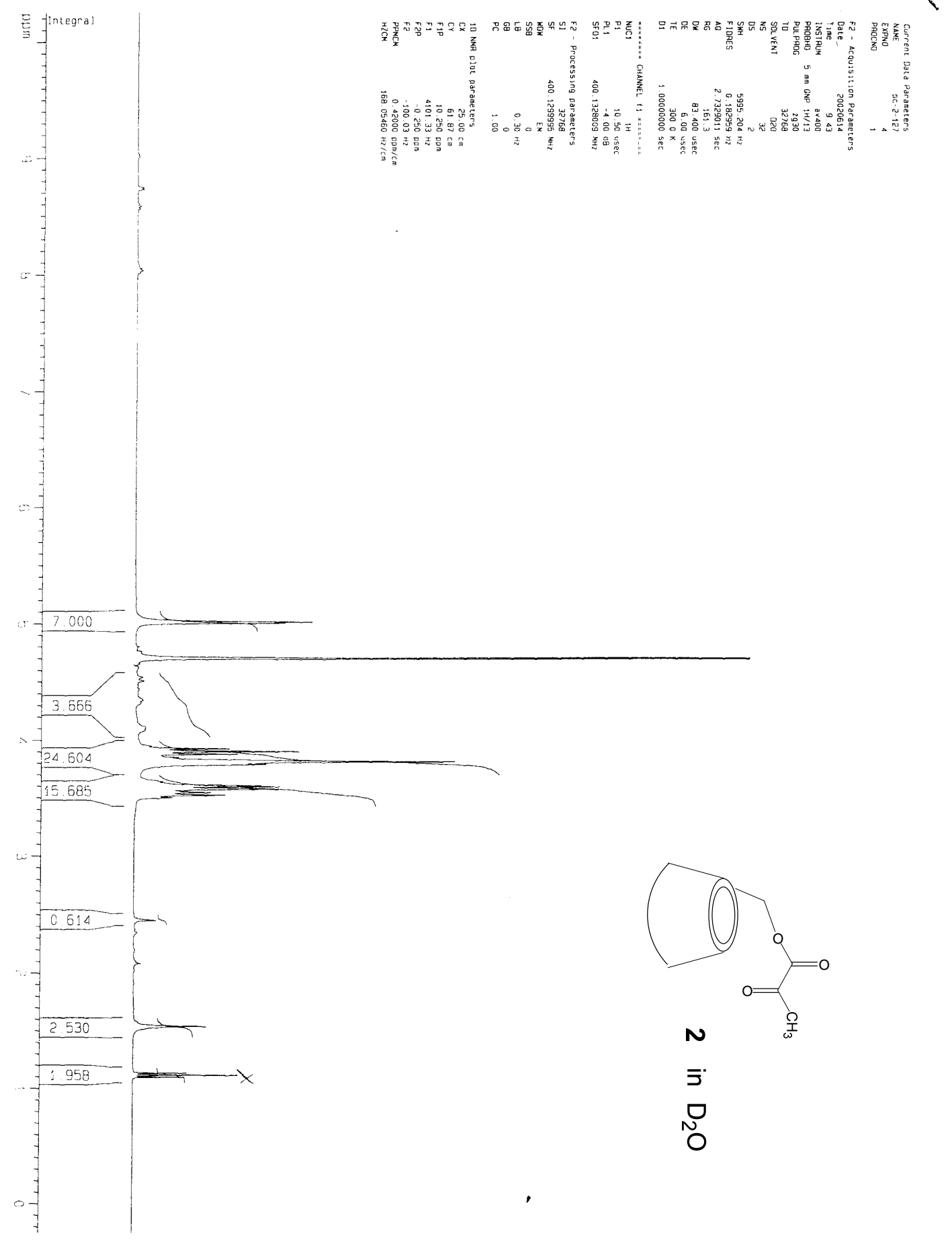



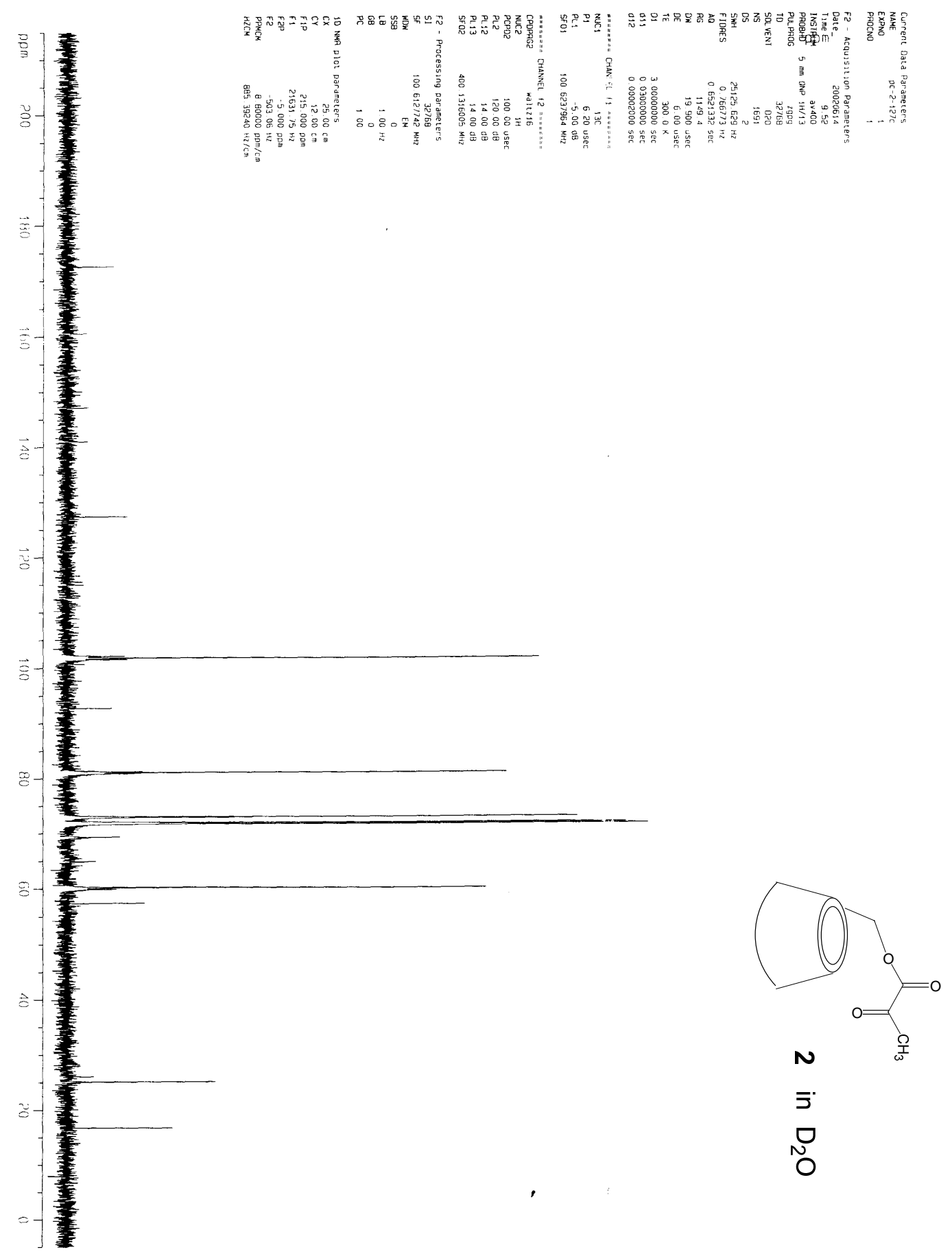


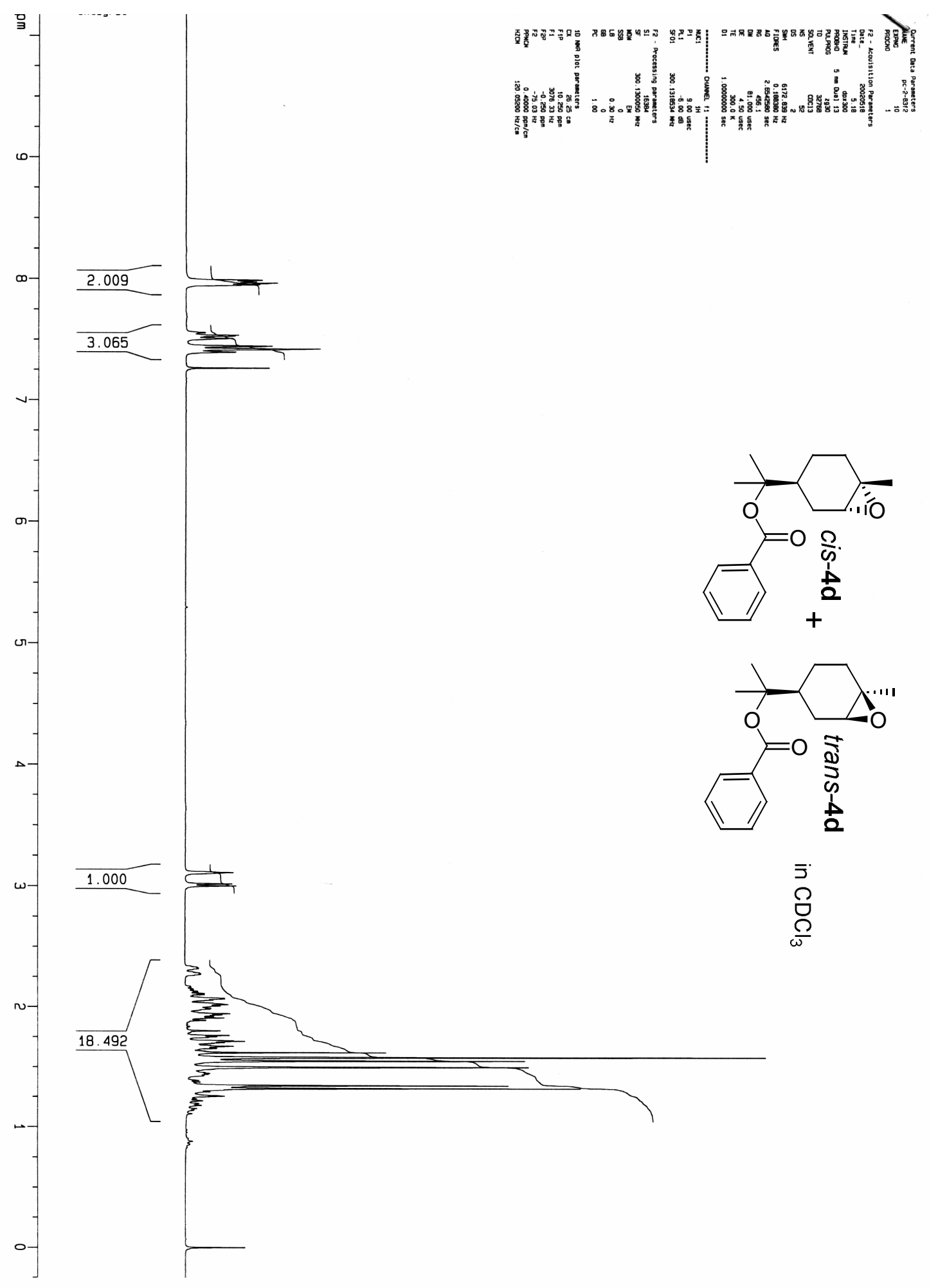




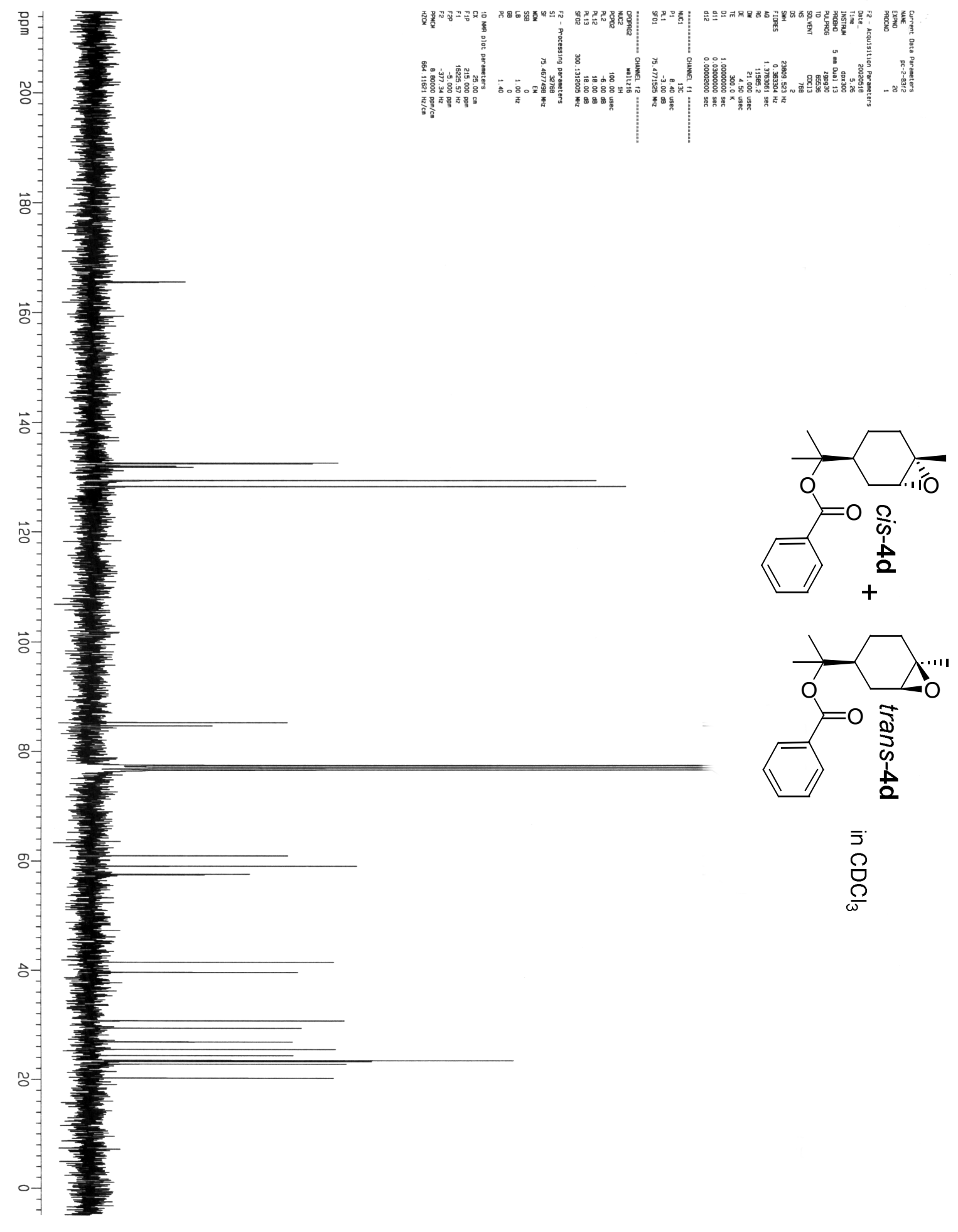




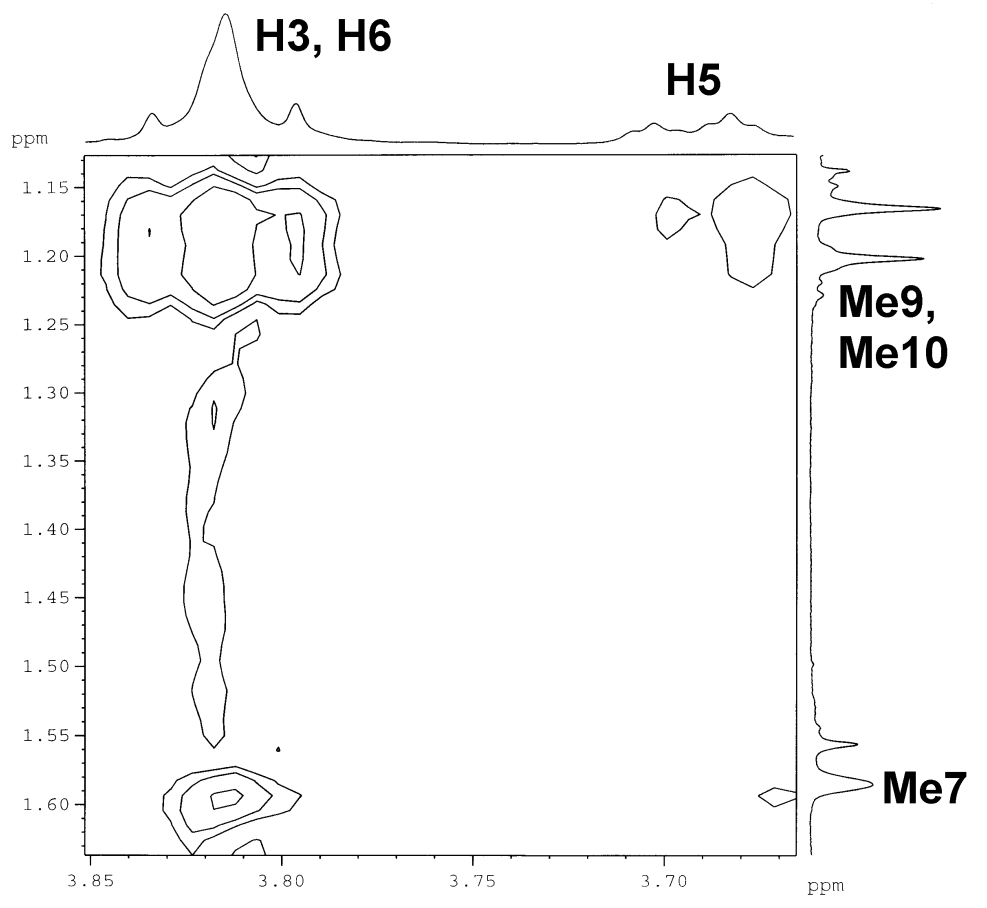

Partial contour plot of $500 \mathrm{MHz}$ ROESY spectrum for binding of $\mathbf{3 b}(5.9 \mathrm{mM})$ to $\mathbf{2}(5.1 \mathrm{mM})$ in $\mathrm{D}_{2} \mathrm{O}$. 